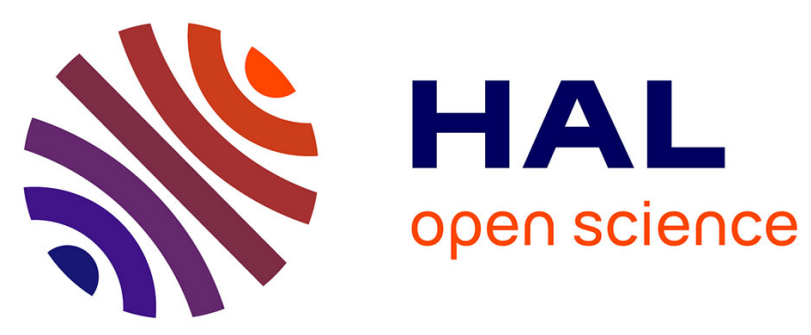

\title{
Perceived Head-Trunk Angle During Microgravity Produced by Parabolic Flight
}

Hadrien Ceyte, Marion Trousselard, Pierre-Alain Barraud, Alain H. Roux, Corinne Cian

\section{- To cite this version:}

Hadrien Ceyte, Marion Trousselard, Pierre-Alain Barraud, Alain H. Roux, Corinne Cian. Perceived Head-Trunk Angle During Microgravity Produced by Parabolic Flight. Aviation, Space, and Environmental Medicine, 2008, 79 (4), pp.420-423. 10.3357/asem.2137.2008 . hal-02003795

\section{HAL Id: hal-02003795 \\ https://hal.science/hal-02003795}

Submitted on 25 Jun 2019

HAL is a multi-disciplinary open access archive for the deposit and dissemination of scientific research documents, whether they are published or not. The documents may come from teaching and research institutions in France or abroad, or from public or private research centers.
L'archive ouverte pluridisciplinaire HAL, est destinée au dépôt et à la diffusion de documents scientifiques de niveau recherche, publiés ou non, émanant des établissements d'enseignement et de recherche français ou étrangers, des laboratoires publics ou privés. 


\title{
Perceived Head-Trunk Angle During Microgravity Produced by Parabolic Flight
}

\author{
Hadrien Ceyte, Marion Trousselard, \\ Pierre-Alain Barraud, Alain Roux, and Corinne Cian
}

Ceyte H, Trousselard M, Barraud P-A, Roux A, Cian C. Perceived head-trunk angle during microgravity produced by parabolic flight. Aviat Space Environ Med 2008; 79:1-4.

Introduction: Neck proprioceptors are essential for orienting the head relative to the trunk. However, it has been shown that the available information about the relationship of gravity to different body parts would augment the clues about their relative orientation. In weightlessness, the absence of relevant body position signals from the otoliths and other inertial graviceptors requires the substitution of other sensory information. The aim of the present study was to investigate the ability of humans to accurately locate the head relative to the trunk in microgravity. Methods: Experiments were conducted during two separate sessions: 1) on Earth and 2) during parabolic flights. Volunteers were asked to adjust a visual rod until it looked parallel to their head or trunk axis in two different segmental configurations: head and trunk aligned or head tilted. Results: There was no effect of microgravity when the head and trunk were aligned. However, when the head was tilted with respect to the trunk, the orientation of the visual rod relative to the head or the trunk (visual egocentric coordinates) was deviated toward the head tilt, although the orientation between the body parts themselves (head-trunk angle) was correctly estimated. Discussion: These results suggested that, in microgravity, the proprioceptive signals from neck muscles seem sufficient to provide accurate head on trunk information. However, the representation of orientation in visual space was modified. This experiment provides evidence for the role of gravity on the visual perception of head- and trunk-based egocentric coordinates.

Keywords: microgravity, egocentric coordinates, proprioceptive cues.

U NDER TERRESTRIAL conditions, the force of gravity provides a constant reference for orientation in unequivocally determining the direction of up and down. The gravitational vector is chosen as a primary reference for self-estimations of body and object orientation relative to the external environment. In weightless conditions of orbital or parabolic flight, personal reports by several astronauts and cosmonauts suggest that they rely on their body longitudinal axis (Z-axis) for the estimation of their actual orientation $(3,11)$. This observation indicates that in the absence of gravity the body may be the default frame of reference (14).

An internal representation of the Z-axis, i.e., a virtual line running from the head to the feet, is used as an egocentric reference frame (7). The processes which give rise to the perceived body Z-axis coordinates are computed through the integration of inputs from multiple sensory sources (visual, proprioceptive, somatosensory, and vestibular) which are continuously reactualized by new body orientation in terrestrial conditions $(2,6)$.
When environmental conditions change, the central nervous system must adapt to the absence of certain sensory signals and reinterpret others (4). In weightlessness, the absence of relevant body position signals from the otoliths and other inertial graviceptors leads to the substitution of other sensory information $(3,14)$. In the absence of environmental visual cues, weightlessness induces a misperception of the Z-axis which is improved by mechanical pressure applied to the torso (3). Moreover, a perceptual deficit of the relative configuration of body parts was reported by some astronauts during spaceflight (10). These verbal reports suggest that proprioceptive information may not be sufficient for an accurate perception of the configuration of body parts in weightlessness.

The aim of the present study was to investigate the ability of humans to accurately locate the head relative to the trunk in microgravity. For this purpose, volunteers were asked to estimate their head or trunk orientation in different segmental configurations. Whereas the neck proprioceptors are essential for orienting the head in relation to the trunk (8), it has been shown that available information about the relationship of gravity to the different body parts can augment the information about their mutual relationship (13). In absence of graviceptive cues, it can be suggested that the head-trunk angle may be misperceived.

\section{METHODS}

The protocol was approved by the ethics committee, CCPRB, which governs and regulates human experimentation in France. Seven volunteers (mean age 32 yr) provided their informed consent to take part in the experiment and passed the equivalent of an Air Force

From the Centre de Recherches du Service de Santé des Armées, La Tronche, France.

This manuscript was received for review in May 2007. It was accepted for publication in December 2007.

Address reprint requests to: Corinne Cian, Centre de Recherches du Service de Santé des Armées, BP 87, 38702 La Tronche Cedex, France; corinnecian@crssa.net.

DOI: 10.3357/ASEM.2137.2008 
Class III medical examination. All volunteers were right handed and had a leading right eye. They had no previous experience with a microgravity environment and were not knowledgeable about the purpose of the experiment. Three of them took medication (a combination of scopolamine and caffeine) before boarding the plane to prevent motion sickness.

\section{Apparatus}

The device used (Fig. 1.) was composed of a cylinder made of white translucent plastic $(70 \mathrm{~cm}$ long, $30 \mathrm{~cm}$ diameter). At the bottom of this device was a metal disk equipped with a black rod ( $30 \mathrm{~cm}$ long and $1 \mathrm{~cm}$ wide). Mounted on the center of the disk, the rod could be rotated $360^{\circ}$ around its central axis by means of a manual joystick. The back of the disk was graduated in degrees (accuracy of $0.5^{\circ}$ ) in order to indicate the rod orientation. At the aperture of the cylinder, the volunteer's head was held in place by a rubber device adjustable to the head size that allowed the orientation of the head to be either maintained upright $\left(0^{\circ}\right)$ or tilted $25^{\circ}$ to the left relative to the trunk axis. The stool on which the volunteers were seated was adjustable so that the rod rotated on their frontal plane about the midpoint of the interocular axis. To ensure the volunteers' integrity, they were held in place by a loose-fitting harness which wrapped the trunk at the level of shoulders and thighs, and by straps which restrained the feet. These attachment systems maintained their body perpendicular to the floor of the airplane. When seated in front of the device, the tunnel and the rod were the only objects visible.

\section{Procedure}

Each volunteer participated in two experimental sessions: 1 ) on the ground ( $1 \mathrm{G}$ ) before flight and 2) during microgravity $(\mu \mathrm{G})$. The microgravity session was carried out during three parabolic flights aboard an Airbus A300 based in Bordeaux, France. Each parabola started from level flight at $1 \mathrm{G}$ and consisted of a 20 -s pull-up at $1.8 \mathrm{G}$, during which the aircraft climbed from 6000 to

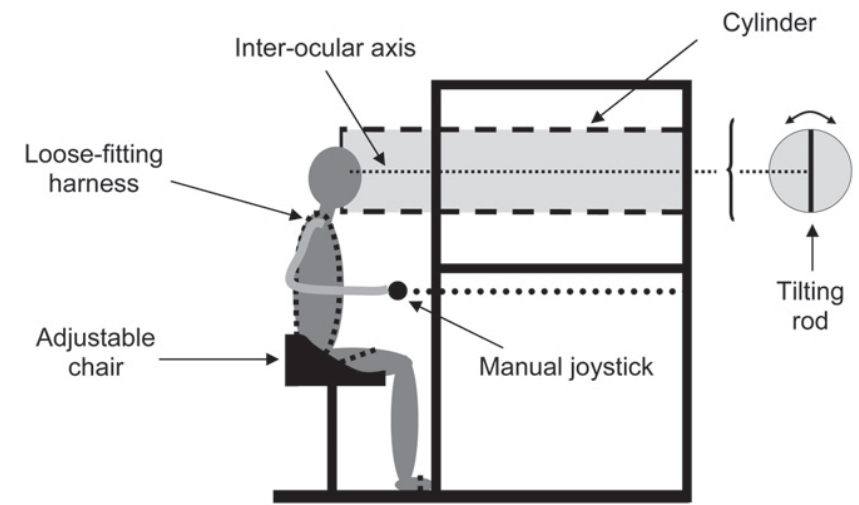

Fig. 1. The device used in the experiment consisted of a horizontal cylinder in which the only visible object was a rod which could rotate around an axis located on the center of the cylinder corresponding to the $\mathrm{X}$-axis on the midpoint of the volunteer's interocular axis. The orientation of the rod could be modified by means of the manual joystick.
$8500 \mathrm{~m}, 20 \mathrm{~s}$ of microgravity obtained over the top of the trajectory, and then a symmetrical 20 -s pull-out at $1.8 \mathrm{G}$ to bring the aircraft back to horizontal flight at the original altitude. There was an interval of approximately 2 min between successive parabolas.

There were 20 trials per session, 10 for the head reference condition and 10 for the trunk reference condition. For each reference condition four trials were conducted with the head and trunk aligned and six trials with the head tilted $25^{\circ}$ to the left side. The order of the different conditions (segmental configuration and reference) was counterbalanced across volunteers.

Once seated in front of the device with the head tilted or upright, the volunteers were instructed to rotate the rod until it looked parallel to their head or trunk axis. The starting position of the rod was tilted $\pm 15^{\circ}$ relative to the objective orientation of the reference (head or trunk axis). The head axis was defined as the virtual 'forehead to chin' axis. The trunk axis was defined as the virtual 'sternal manubrium to pubis' axis. The volunteers signaled verbally that they had completed their adjustment and closed their eyes. Thereafter the rod was rotated to the next preset position. The volunteers opened their eyes and conducted a second trial in the same segmental configuration and with the same instruction (head or trunk reference). At the end of the second trial, their head was removed from the device. This procedure allowed them to perform two trials during the same parabola in the microgravity session. No time constraint was imposed during the adjustment phase, but the volunteers were encouraged to respond in a timely fashion. No feedback was given regarding the performance.

\section{Data Collection}

The objective orientation of the trunk was $0^{\circ}$ on the graduated disk, whereas the objective orientation of the head was either $0^{\circ}$ when aligned with the trunk or $25^{\circ}$ when tilted. Two parameters were analyzed. The first, the perceived head-trunk angle, was the mean angular difference between head and trunk adjustments in each segmental configuration; angles were arbitrarily considered to be positive if the head was perceived to be tilted to the right relative to the trunk and negative if the head was perceived to be tilted left. $0^{\circ}$ and $-25^{\circ}$ were considered as the objective angle for aligned and head tilted, respectively. The second parameter, egocentric coordinates, were the mean angular deviation (degrees) between the objective orientation of the head or trunk axis and the volunteer's response (orientation of the rod). Errors were arbitrarily considered from the volunteer's perspective to be positive if the upper pole of the rod deviated to the right from the objective orientation of the head or trunk axis and negative if the rod deviated to the left.

\section{RESULTS}

None of the volunteers exhibited symptoms of motion sickness before or during the experiment. They 
did not report any difficulties in completing the experimental tasks during the terrestrial gravity or microgravity sessions. All volunteers stated that they were not aware of their orientation relative to the aircraft when changing the settings during the microgravity phase.

Results demonstrated that the perceived head-trunk angles were of the same magnitude for $1-G$ and $\mu \mathrm{G}$ sessions in the aligned (paired $t$-test, $P>0.05 ; \operatorname{Mean}(\mathrm{M})=$ $-0.54^{\circ}, \mathrm{SD}=1.46 ; \mathrm{M}=1.06^{\circ}, \mathrm{SD}=1.8$ in $1 \mathrm{G}$ and $\mu \mathrm{G}$, respectively) and head tilted conditions (paired $t$-test, $P>0.05 ; \mathrm{M}=-24.6^{\circ}, \mathrm{SD}=3.2 ; \mathrm{M}=-20.2^{\circ}, \mathrm{SD}=5.6$ in $1 \mathrm{G}$ and $\mu \mathrm{G}$, respectively). Moreover, there was no systematic error of perceived head-trunk angles during the gravitational sessions and segmental conditions (aligned condition: $t$-test against $0^{\circ}$, which corresponded to the objective tilt of the head, $P>0.05$; tilted condition: $t$-test against $-25^{\circ}$, which corresponded to the objective tilt of the head, $P>0.05$ ).

A 3-way analysis of variance, 2 gravitational sessions $(1 \mathrm{G}$ vs. $\mu \mathrm{G}) \times 2$ segmental configurations (aligned condition vs. head tilted condition) $\times 2$ reference conditions (head and trunk), with repeated measures on the three factors were applied to egocentric coordinates. A post hoc analysis (Newman-Keuls) was performed when $P<0.05$. Results showed main effects of gravitational session $[\mathrm{F}(1,6)=21.12, P<0.05]$ and segmental configuration $[\mathrm{F}(1,6)=18.9, P<0.05]$, but no main effect of the reference condition $(P>0.05)$. There was also a significant interaction of the gravitational session and segmental configuration factors $[\mathrm{F}(1,6)=66.74, P<0.05]$. When the head was tilted relative to the trunk, the microgravity session induced a significant left deviation of the head and trunk orientations, whereas there was no ef-

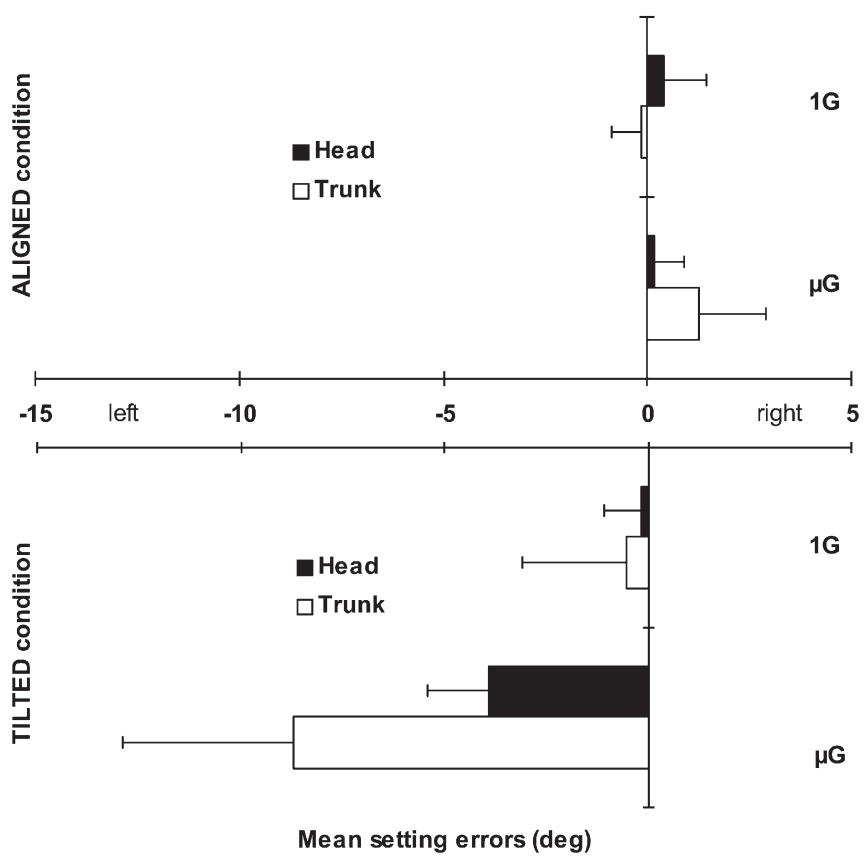

Fig. 2. Mean setting errors (degrees) for the aligned and head tilted conditions under terrestrial gravity $(1 \mathrm{G})$ or in the microgravity $(\mu \mathrm{G})$ environment. Error bars indicate the SD. fect of microgravity when the head and trunk were aligned (Fig. 2.).

\section{DISCUSSION}

This study was designed to investigate the effect of microgravity on the ability to locate the head relative to the trunk. The main findings of this study showed that 1) the head-trunk angle was similarly perceived in $1 \mathrm{G}$ and $\mu \mathrm{G}$, but 2) that the accuracy in visual orientation of objects relative to these body segments (egocentric coordinates) was disturbed in microgravity when the head and the trunk were not aligned. With head and trunk aligned, the absence of systematic deviation of the head and trunk axes suggests that the Z-axis was correctly perceived. Our data seems to contradict the findings of Clément et al. (3), which showed that the perception of the $\mathrm{Z}$-axis is less accurate in the absence of any graviceptive cues. However, it is in agreement, in part, with one of their results that indicated that as soon as mechanical pressures are applied on the body surface, the perception of the Z-axis is improved (3). Indeed, in the present experiment, the volunteers were held in place by an attachment system which induced some somatosensory stimulation on the contact surface of their body.

When the head was tilted, the perception of the orientation of the head relative to the trunk (head-trunk angle) also remained accurate whatever the gravity environment. This result suggests that the presence of pressure points and/or the neck proprioceptive cues are adequate to estimate the configuration of body parts in microgravity. However, the existence of a neural representation of the gravity vector which is developed and enhanced with spatial experience has been suggested (9). This internal representation of gravity may be available during the short period of weightlessness and may be used to perceive the different body parts and their mutual relationship (13). Whatever the explanation considered, none of them seem sufficient to perceive egocentric coordinates accurately when the head was tilted relative to the trunk. Indeed, perceived orientation of both head and trunk axes were deviated toward the head tilt.

Perceptual deviations of egocentric coordinates remain unclear. Ocular counter-rolling cannot account for this observed difference between aligned and tilted conditions in microgravity. Indeed, in microgravity, the ocular counter-rolling induced by static head tilt to the right or left did not differ significantly from those obtained when the volunteer's head was upright (5). Similar deviations of egocentric coordinates have been observed on Earth in restrained volunteers with head and trunk aligned who were exposed to a neck proprioceptive stimulation by vibration. A head-trunk dissociation is normally associated with a vestibular and proprioceptive stimulation. However, in absence of change in vestibular inputs, the perception of a change in muscle length induced by this proprioceptive stimulation resulted in a virtual roll displacement of egocentric coordinates toward the vibrated side without modification 
of the perceived head-trunk angle (1). It was stated that this vestibulo-proprioceptive conflict favors the proprioceptive signal (1). These data were explained by the existence of a proprioceptive chain (12), which results from the common processing of various feedback arising in different muscles which are stimulated together in a given segmental configuration or orientation. Applying stimulation (i.e., vibration) at any level in the proprioceptive chain could modify the internal representation of the whole body in the direction of this stimulation (12). We suggest that, in weightlessness, the unusual patterns of vestibular and proprioceptive stimulation relative to a head to trunk tilt, i.e., the otolith information being missing in microgravity, also leads to a visual perception mainly determined by proprioceptive input. Consequently, the perception of egocentric coordinates deviates in the direction of the proprioceptive stimulation of the neck.

The assessment of the relationship between external objects and the body of the observer is a fundamental human ability. Visual egocentric coordinates constitute one basis of the oriented behavioral organization toward the extracorporal space. This ability is disrupted in microgravity, providing evidence for the role of gravity on the perception of head- and trunk-based egocentric coordinates. Human activities often involve sensing body orientation in space. In microgravity, the perceived Z-axis remained the only available reference for spatial orientation. Free-floating humans can feel disoriented in microgravity due to a misperception of the Z-axis coordinates.

\section{ACKNOWLEDGMENTS}

The present experiment was carried out in the A300 0-G aircraft during the $55^{\text {th }}$ parabolic flight campaign organized by the European Space Agency (ESA). The authors thank Dr. Robert Carter for his help with the English text.

\section{REFERENCES}

1. Ceyte H, Cian C, Nougier V, Olivier I, Roux A. Effects of neck muscles vibration on the perception of the head and trunk midline position. Exp Brain Res 2006; 170:136-40.

2. Ceyte H, Cian C, Nougier V, Olivier I, Trousselard M. Role of gravity-based information on the orientation and localization of the perceived body midline. Exp Brain Res 2007; 176:504-9.

3. Clément G, Arnesen TN, Olsen MH, Sylvestre B. Perception of longitudinal body axis in microgravity during parabolic flight. Neurosci Lett 2007; 413:150-3.

4. Clément G, Berthoz A. Influence of gravity on the processing of information by the central nervous system. In: Oser $\mathrm{H}$, Guyenee TD, eds. Proceedings of the $5^{\text {th }}$ European Symposium on 'Life Sciences Research in Space.' Arcachon, France: ESA SP-366 European Space Agency; 1994:333-8.

5. Diamond SG, Markham CH, Money KE. Instability of ocular torsion in zero gravity: possible implications for space motion sickness. Aviat Space Environ Med 1990; 61:899-905.

6. Gurfinkel VS, Lestienne F, Levik YuS, Popov KE. Egocentric references and human spatial orientation in microgravity. Exp Brain Res 1993; 95:339-42.

7. Jeannerod M, Biguer B. Référence égocentrique et espace représenté (Egocentric reference and representation of space). Rev Neurol (Paris) 1989; 145:635-9.

8. Maurer C, Kimmig H, Trefzer A, Merger T. Visual object localization through vestibular and neck inputs. I: localization with respect to space and relative to the head and trunk midsagittal planes. J Vestib Res 1997; 7:119-35.

9. Merfeld DM, Zupan LH, Gifford CA. Neural processing of gravitoinertial cues in humans. II: influence of the semicircular canals during eccentric rotation. J Neurophysiol 2001; 85:1648-60.

10. Money KE, Cheung BS. Alterations of proprioceptive function in the weightless environment. J Clin Pharmacol 1991; 31:1007-9.

11. Oman CM, Lichtenberg BK, Money KE. Space motion sickness monitoring experiment: Spacelab 1. In: Crampton GH, ed. Motion and space sickness. Boca Raton, FL: CRC Press; 1990: 217-46.

12. Roll JP, Roll R. From eye to foot: a proprioceptive chain involved in postural control. In: Amblard B, Berthoz A, Clarac F, eds. Posture and gait: development, adaptation and modulation. Paris: Elsevier Science Publisher BV (Biomedical Division); 1988:155-64.

13. Templeton WB. The role of gravitational cues in the judgment of visual orientation. Percept Psychophys 1973; 14:451-7.

14. Young LR. Vestibular reactions to spaceflight: human factors issues. Aviat Space Environ Med 2000; 71(9, Suppl.)A100-4. 